1

2

3

4

5

6

7

8

9

10

\title{
Hierarchical Porous Nitrogen-Doped Carbon by Pickering HIPEs Technique: Synthesis and Application in HMF Production
}

Bing Li ${ }^{\mathrm{a}}$, Yao Chen ${ }^{\mathrm{b}, ~ *}$, Wen Guan ${ }^{\mathrm{a}}$, Yanan Wei ${ }^{\mathrm{a}}$, Yunlei Zhang ${ }^{\mathrm{a}, ~ *}$, Yongsheng Yan ${ }^{\mathrm{a}}$

a Institute of Green Chemistry and Chemical Technology, School of Chemistry and

Chemical Engineering, Jiangsu University, Zhenjiang, P. R. China

${ }^{b}$ School of the Environment and Safety, Jiangsu University, Zhenjiang, P. R. China

*Corresponding author. Email: chenyao1390@ujs.edu.cn (Y. Chen)

zylff035@126.com (Y. Zhang);

Tel: +8651188830099 Fax: +8651188830099 
Synthesis of acid and base catalyst: Firstly, in order to obtain water-in-oil Pickering HIPEs, we adjusted the hydrophilicity of the obtained SBA-15. The steps of grafting amino on the surface of SBA-15 were as follows: Toluene $(285 \mathrm{~mL}), \mathrm{SBA}-15(1.9 \mathrm{~g})$ and $\mathrm{KH}-550(10 \mathrm{~mL})$ were added into a three-mouth flask to disperse evenly. The reaction was placed in an oil bath at $110^{\circ} \mathrm{C}$ and refluxed under a nitrogen atmosphere for $12 \mathrm{~h}$. The products were then collected, washed with toluene, centrifuged, and dried in a vacuum at $60{ }^{\circ} \mathrm{C}$. The amino-grafted SBA-15(1.0 g) was suspended in the mixture of OA and chlorine (1:4 M ratio), stirred for $3.0 \mathrm{~h}$, and oA-SBA-15 was extracted from the solution by methanol precipitation and dried in an oven at $60{ }^{\circ} \mathrm{C}$.

Secondly, water in oil (W/O) Pickering HIPEs template was formed by using oleic acid modified SBA-15 particles obtained in the previous step as stabilizer. A detailed procedure for acid catalyst is as follows: OA-SBA-15 (1.5 wt \% of oil phase mass), AIBN (0.06 g) and polymer $2296(0.13 \mathrm{~g})$ were dissolved in the mixture of DVB $(3.0 \mathrm{~mL})$ and toluene $(2.0 \mathrm{~mL})$ to form a continuous external phase under mild stirring. Afterwards, under continuous stirring, the inner water phase $(26.7 \mathrm{~mL}$ deionized water) was added to the oleic phase dropwise to obtain stable W/O HIPEs. Pickering HIPEs were transferred to a sealed glass mold, polymerized in a $70{ }^{\circ} \mathrm{C}$ water bath for more than 12 $\mathrm{h}$, and then washed with acetone through Soxhlet extraction for more than $48 \mathrm{~h}$ to remove impurities. The resultant polymer foams were dried to a constant weight in a drying oven at $60^{\circ} \mathrm{C}$. Under the condition of nitrogen atmosphere, the samples were heat-treated for $2.0 \mathrm{~h}$ at a heating rate of $5.0^{\circ} \mathrm{C}$ $\min ^{-1}$, The system temperature was $600^{\circ} \mathrm{C}$. The synthesis procedure of base catalyst was same as acid catalyst except for replacing DVB with a mixture of DVB (3.0 mL) and 1-vinyl imidazole (6.0 $\mathrm{mL}$ ), the final synthesized base catalyst was named NC-700.

Finally, the acid catalyst prepared in the previous step was sulfonated. In general, acid catalyst $(5.0 \mathrm{~g})$ was added into $50 \mathrm{~mL}$ concentrated sulfuric acid $(98 \%)$, protected by nitrogen for $0.5 \mathrm{~h}$, and heated at $180^{\circ} \mathrm{C}$ for $5.0 \mathrm{~h}$. Then the sulfonated mixture was cooled to room temperature and diluted with deionized water to form a black sediment, filtered and repeatedly washed with a large amount of deionized water until the filtrate was neutral. The obtained samples were dried in a vacuum at $60^{\circ} \mathrm{C}$ for $48 \mathrm{~h}$, the final synthesized acid catalyst was named SC-700. 
(a) $\quad \begin{aligned} & \text { SBA-15 } \\ & \approx 45.2^{\circ}\end{aligned}$

(b)

OA-SBA-15

$\approx 115.2^{\circ}$

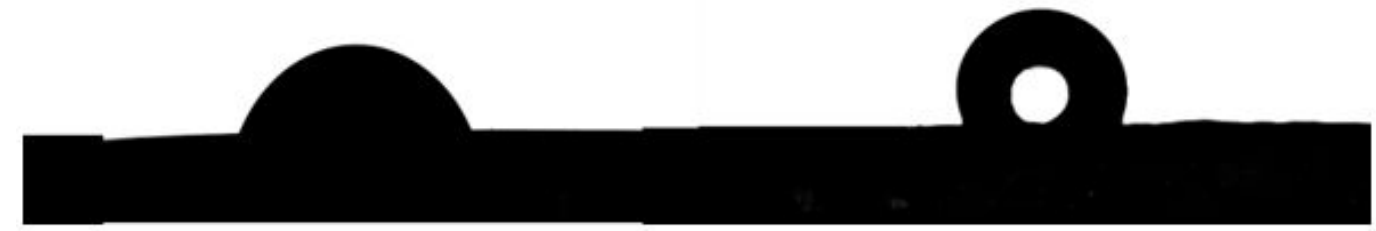

Figure. S1 Water contact angles of SBA-15 and 1:4 OA-SBA-15.
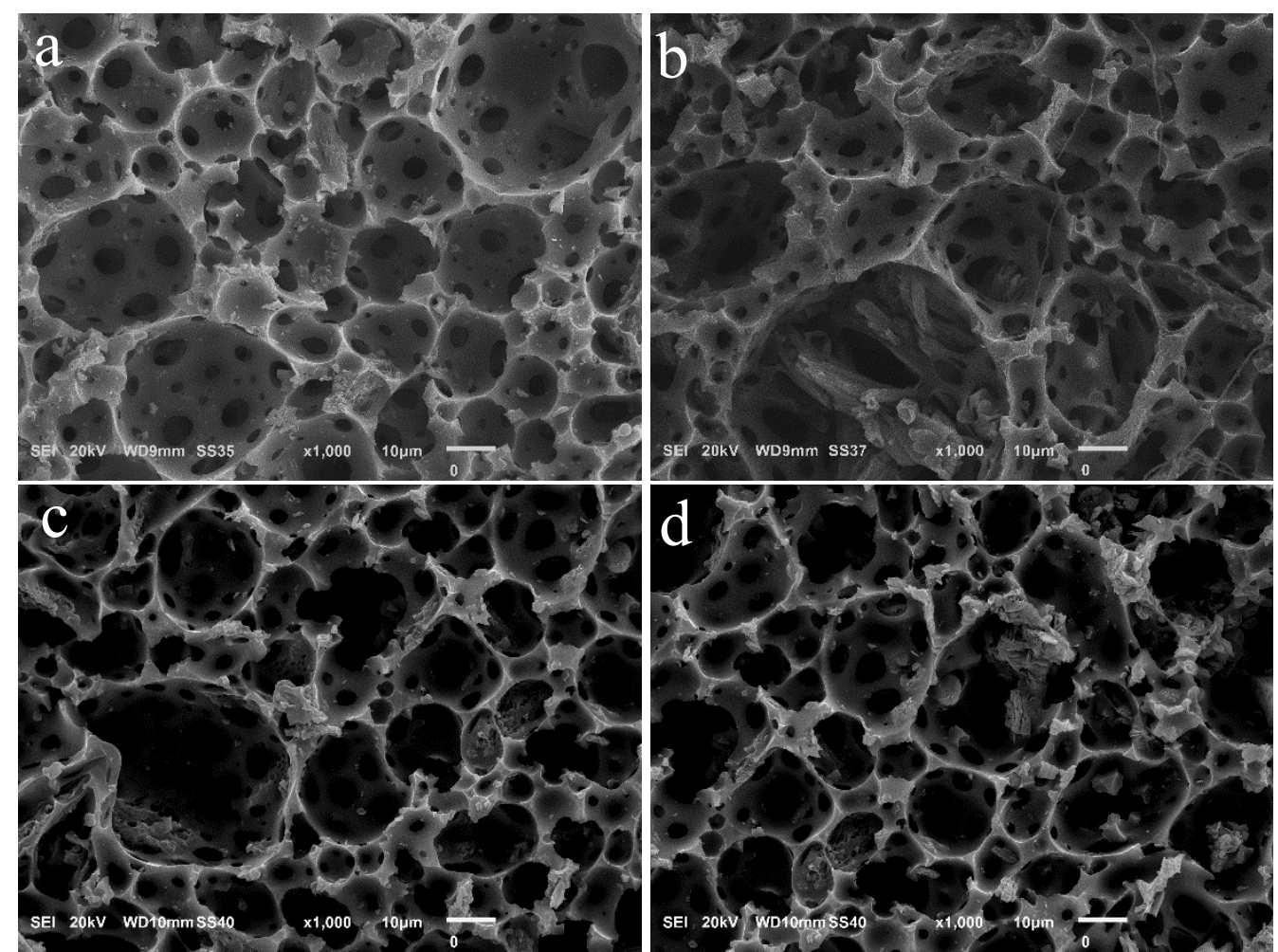

Figure. S2 (a-d) SEM images of SNC2-700, SNC3-700, NC-700, SC-700. 


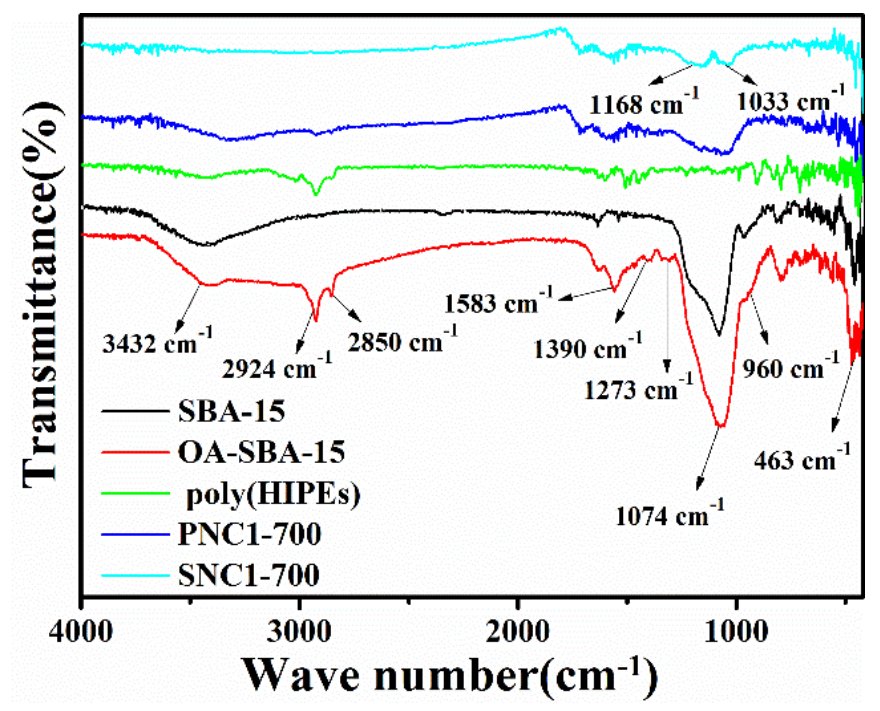

41 Figure. S3 FT-IR spectra of SBA-15, OA-SBA-15, poly (HIPEs), PNC1-700 and SNC1-700.

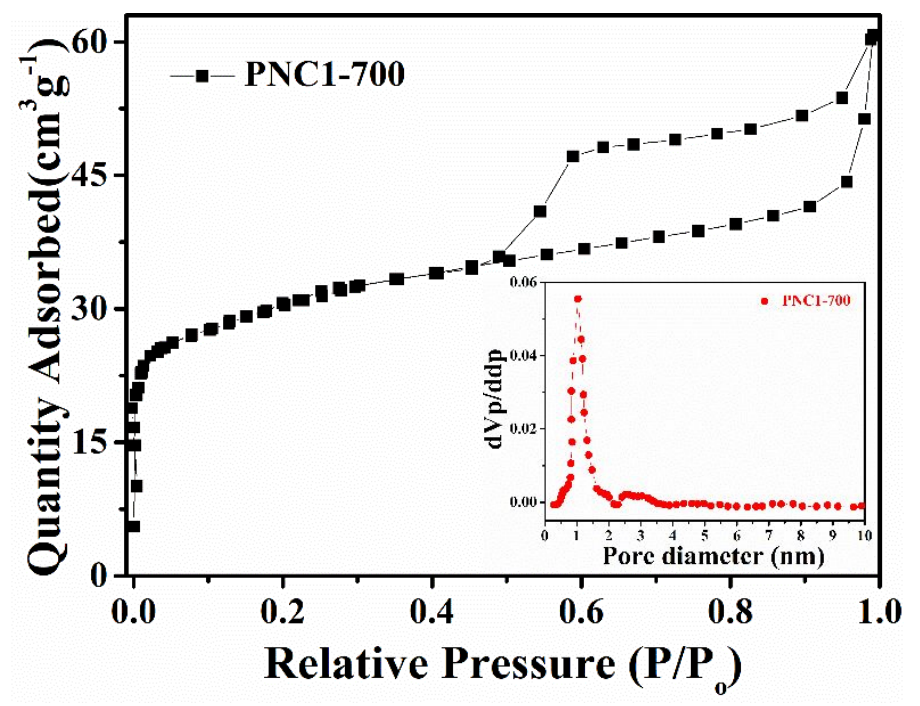

42 Figure. S4 Nitrogen adsorption-desorption isotherms and pore size distributions of PNC1-700.

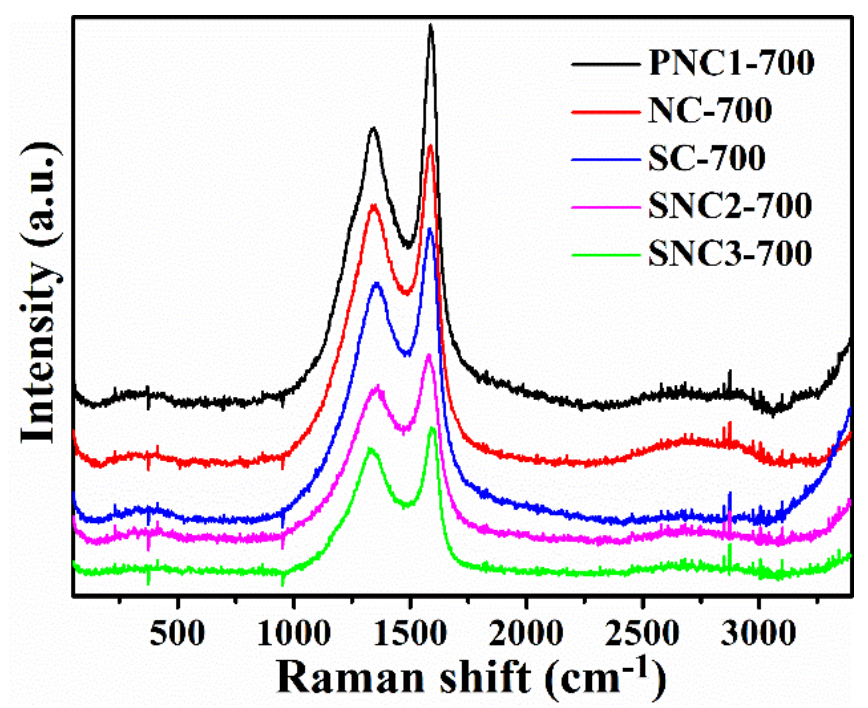

43 Figure. S5 Raman spectra of PNC1-700, SNC2-700, SNC3-700, SC-700, NC-700 materials at 44 different pyrolysis temperatures. 
(a)

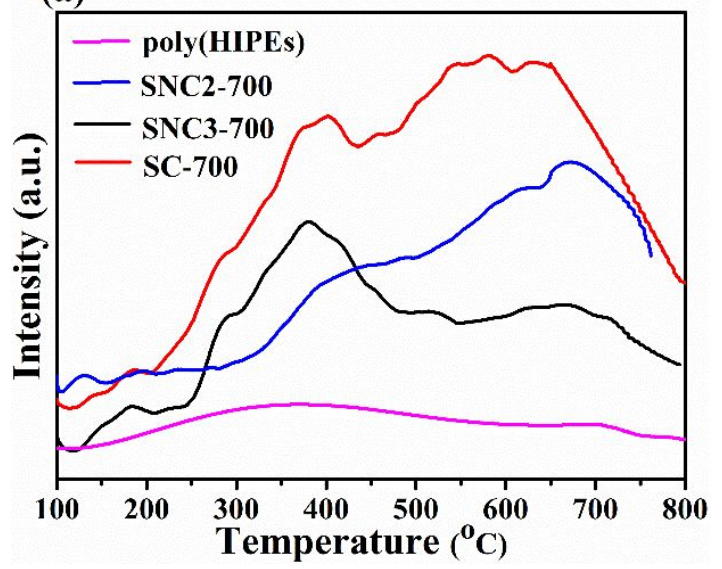

(b)

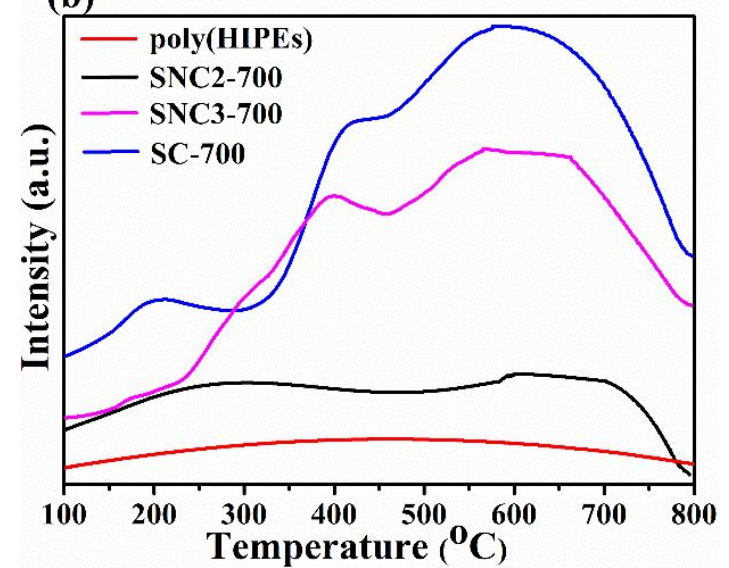

Figure. $\mathbf{S 6} \mathrm{CO}_{2}$-TPD (a) and $\mathrm{NH}_{3}-\mathrm{TPD}$ (b) curves of catalysts.

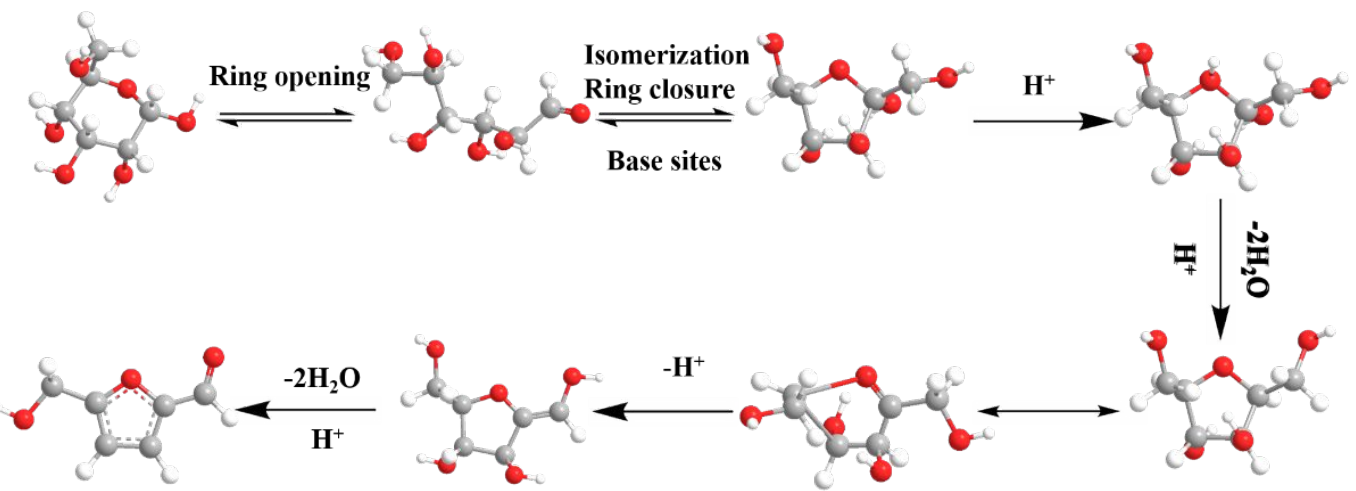

46 Figure. S7 The plausible catalytic scheme for the conversion of glucose into HMF over our developed acid-base bi-functional catalyst. 